\title{
Image guided radiation therapy boost in combination with high-dose-rate intracavitary brachytherapy for the treatment of cervical cancer
}

\author{
Xianliang Wang, MSc, Jie Li, MSc, Pei Wang, MSc, Ke Yuan, MSc, Gang Yin, PhD, Bin Wan, MSc \\ Department of Radiation Oncology, Sichuan Cancer Hospital, Chengdu, China
}

\begin{abstract}
Purpose: The purpose of this study was to demonstrate the dosimetric and clinical feasibility of image guided radiation therapy (IGRT) combined with high-dose-rate (HDR) intracavitary brachytherapy (ICBT) to improve dose distribution in cervical cancer treatment.

Material and methods: For 42 cervical cancer patients, magnetic resonance imaging (MRI) scans were acquired after completion of whole pelvic irradiation 45-46 Gy and 5 fractions of B + I (ICBT + IGRT) treatment were subsequently received. The high risk clinical target volume (HRCTV), intermediate risk clinical target volume (IRCTV), bladder, rectum, and sigmoid were contoured on the computed tomography (CT) scans. The total planning aim doses for HRCTV was $\mathrm{D}_{90 \%}$ $>85 \mathrm{~Gy}$, whilst constraints for rectum and sigmoid were $\mathrm{D}_{2 \mathrm{cc}}<75 \mathrm{~Gy}$ and $\mathrm{D}_{2 \mathrm{cc}}<90 \mathrm{~Gy}$ for bladder in terms of an equivalent dose in 2 Gy (EQD2) for external beam radiotherapy (EBRT) and brachytherapy boost. The IGRT plan was optimized on top of the ICBT dose distribution. A dosimetric comparison was made between B + I and optimized ICBT (O-ICBT) only.

Results: The mean $\mathrm{D}_{90 \%}$ of HRCTV was comparable for B + I and O-ICBT $(p=0.82)$. For B + I plan, HRCTV $\mathrm{D}_{100 \%}$, IRCTV $D_{100 \%}$, and IRCTV $D_{90 \%}$ were significantly increased by a mean of $10.52 \mathrm{~Gy}, 5.61 \mathrm{~Gy}$, and 2.70 Gy, respectively $(p<0.01)$. The $\mathrm{D}_{2 \mathrm{cc}}$ for bladder, rectum, and sigmoid were lower by a mean of $21.36,6.78$, and 10.65 Gy, respectively $(p<0.01)$. The mean rectum V60 Gy value over 42 patients was almost the same for both techniques but for bladder and sigmoid B + I had higher V60 Gy mean values as compared with the O-ICBT.

Conclusions: $\mathrm{B}+\mathrm{I}$ can improve dose distribution in cervical cancer treatment; it could be useful for tumors extended beyond the reach of intracavitary/interstitial brachytherapy (IC/ISBT) or for centers that are inexperienced or illequipped with IC/ISBT techniques. Additional confirmatory prospective studies with larger numbers of patients and longer follow-up are required to validate the durability.
\end{abstract}

J Contemp Brachytherapy 2016; 8, 2: 122-127 DOI: $10.5114 /$ jcb.2016.59282

Key words: cervical cancer, HDR brachytherapy, IGRT.

\section{Purpose}

Conventional radiotherapy for cervical cancer combines external beam radiotherapy (EBRT) with a boost of brachytherapy. The efficacy of a brachytherapy boost has been well recognized for cervical cancer $[1,2,3,4]$. However, tumors with significant residual disease or unfavorable topography after EBRT may still represent a therapeutic challenge for conventional brachytherapy. Studies have demonstrated that the pear-shaped dose distribution of intracavitary brachytherapy (ICBT) often fails to cover the entire target volume, especially for patients with late stage disease $[5,6]$. In these cases, it can be difficult to deliver the prescribed dose to the target with brachytherapy because of the inverse square law and the need to respect organs at risk (OARs) constraints $[7,8]$. This can lead to lower local control rates.
There are currently three main techniques used to improve local control and survival for patients with a large cervical cancer: optimization of the pear-shaped isodoses, external beam boost, and interstitial brachytherapy (ISBT). Optimization of the pear-shaped isodose is the easiest and simplest approach for improving tumor coverage [9], however, the finite number of source positions is a limiting factor for the optimization results. It is difficult for optimization alone to cover a large or an asymmetrical tumor without significantly increasing the dose to the OARs. External beam boost performed with midline block fields has been used to boost parametrial extension in patients in whom the contribution from ICBT was insufficient $[10,11]$. However, its effectiveness is poorly documented [12], and there is no strong evidence to validate its routine use. Interstitial brachytherapy is helpful
Address for correspondence: Pei Wang, MsC, Department of Radiation Oncology, Sichuan Cancer Hospital, No.55, the 4th Section, Renmin South Road, Chengdu, 610041, China, phone: +8613881907458 , 028 85420659, 凶 e-mail: dengwangpei@163.com
Received: 23.10 .2015

Accepted: 15.02 .2016

Published: 27.04.2016 
in patients with bulky or an obliterated endocervical canal, vaginal spread disease; it can be combined with ICBT by placing needles in the parametrial region or in large tumor. Intracavitary/interstitial brachytherapy (IC/ISBT) can achieve good target coverage and is currently recommended as a standard $[13,14,15,16]$.

In the present study, we provided an alternative technique to boost the parametrial region or large unfavorable tumor. We integrated ICBT with a simultaneous image guided radiation therapy (IGRT) boost to improve target dose coverage and spare of the OARs. We abbreviated this technique to B + I (ICBT + IGRT). The dosimetric and clinical feasibility of this technique was demonstrated A dosimetric comparison was made between $\mathrm{B}+\mathrm{I}$ and optimized ICBT (O-ICBT) only. B + I technique could be useful for tumors extended beyond the reach of IC/ISBT or for centers that are inexperienced or ill-equipped with IC/IS BT techniques.

\section{Material and methods}

Forty-two patients with large or topographically unfavorable tumors were included in this study. The patients were 39-63 years of age at the time of treatment (median 49). All tumors were squamous cell carcinomas, consisted of FIGO (International Federation of Gynecology and Obstetrics) stage IIB-IVA (18 IIB, 7 IIIA, 11 IIIB, 6 IVA).

Magnetic resonance imaging (MRI) scans were acquired after whole pelvic EBRT 45-46 Gy, and subsequently 5 fractions of $\mathrm{B}+\mathrm{I}$ radiation boost were performed based on the MRI and gynecologic examination. At time of brachytherapy, the median volumes of high risk clinical target volume (HRCTV) and intermediate risk clinical target volume (IRCTV) for the 42 cases were $62.88 \pm 21.94 \mathrm{~cm}^{3}$ and $145.15 \pm 36.36 \mathrm{~cm}^{3}$, respectively.

For each fraction, a bladder catheter was used to control bladder filling and a Fletcher applicator (Elekta AB, Stockholm, Sweden) was inserted. The applicator was subsequently fixed to a board, which can slide between the mobile bed, the treatment couch, and the computed tomography (CT) couch. A vacuum bag and a thermoplastic mask were used to fix the patient position and then a CT scan was obtained. There was no image fusion between CT and previous MRI scans because of organ deformations. The targets (HRCTV and IRCTV) were identified from CT and delineated by the radiation oncologist on the CT scans. The OARs (bladder, rectum, and sigmoid) were contoured according to the framework of the international study on MR-guided brachytherapy in locally advanced cervical cancer (EMBRACE) (see http:// www.embracestudy.dk). No margins were added to targets and OARs, since the setup uncertainties and internal motions were minimal.

The total EBRT and brachytherapy boost doses were evaluated in terms of equivalent dose in 2 Gy fractions (EQD2), using $\alpha / \beta=3$ Gy for OARs and $\alpha / \beta=10$ Gy for targets. We define Dx (or Dx\%) as the dose expressed in Gray (Gy) received by $\mathrm{x}$ volume in cc (or $\mathrm{x} \%$ of the total volume), and Vy Gy the volume expressed in percentage that receive y Gy. The treatment planning aim was to ob- tain $\mathrm{D}_{90 \%}>85$ Gy for HRCTV and $\mathrm{D}_{90 \%}>75$ Gy for IRCTV from EBRT and B + I boost. Dose volume constraints for OARs were $\mathrm{D}_{2 \mathrm{cc}}<90 \mathrm{~Gy}$ for the bladder and $\mathrm{D}_{2 \mathrm{cc}}<75 \mathrm{~Gy}$ for rectum and sigmoid. It was assumed that the $D_{2 c c}$ of all three OARs were exposed to the full EBRT dose of 45 Gy. We can calculate physical dose objectives and constraints per fraction by the equation [17]:

$$
E Q D 2=n d\left(1+\frac{d}{\alpha / \beta}\right) /\left(1+\frac{2}{\alpha / \beta}\right)
$$

where $n$ is the number of fraction, $d$ is the dose per fraction, and $\alpha / \beta$ is the $\alpha / \beta$-ratio of the organ concerned. The calculated physical dose objectives were 6 Gy per fraction for HRCTV and 5 Gy per fraction for IRCTV, while the calculated $\mathrm{D}_{2 \mathrm{cc}}$ OARs constraints were $5.4 \mathrm{~Gy}$ per fraction for bladder and 4.2 Gy per fraction for rectum and sigmoid. In clinical practice, bladder $D_{2 c c}$ was optimized less than $5 \mathrm{~Gy}$, since we wanted bladder $\mathrm{D}_{2 \mathrm{cc}}$ lower than IRCTV dose objective.

The ICBT treatment plan was performed with the Oncentra ${ }^{\circledR}$ Brachy V4.3 treatment planning system (Elekta $\mathrm{AB}$, Stockholm, Sweden) and the applicator reconstruction was done according to the GEC-ESTRO (Groupe Européen de Curiethérapie - European Society for Radiotherapy and Oncology) guidelines [18]. For B + I plan, the prescription of the ICBT plan was 5 Gy per fraction and 3D graphical optimization was performed with the aim of optimizing the dose coverage of the target, while not reaching the dose constraint for OARs (5 Gy for bladder and 4 Gy for rectum and sigmoid) in order to allow an additional dose contribution from the IGRT boost. For O-ICBT plan, inverse planning simulated annealing (IPSA) optimization was used to optimize dose distribution. The planning aim was to obtain at least 85 Gy for $\mathrm{D}_{90 \%}$ in HRCTV.

For B + I plan, the target dose coverage was always limited by the dose constraint of the OARs. If a sufficient dose to the target could not be achieved, a seven or nine field IMRT plan was produced with the Oncentra ${ }^{\circledR}$ External Beam V4.3 treatment planning system (Elekta AB, Stockholm, Sweden). The IMRT plan was optimized on top of the ICBT plan using an inverse dose optimization tool, which allows the use of dose volume histograms (DVHs) constraints on the total dose of ICBT and IMRT. The fields entrances were different in order to avoid a highway of dose through the patient and no upper dose constraint to targets was used when optimizing the boost plan.

The B + I and O-ICBT plan were evaluated three dimensionally to determine the targets dose coverage and the sparing of OARs. $\mathrm{D}_{100 \%}, \mathrm{D}_{90 \%}$, and $\mathrm{D}_{50 \%}$ were used for targets comparison, while $\mathrm{D}_{2 \mathrm{cc}}$ and V60 Gy were considered for OARs. V60 Gy represented the volume irradiated to more than $60 \mathrm{~Gy}$ (EQD2) by the accumulated dose from EBRT and brachytherapy boost. The boost plans physical isodose level was 2.65 Gy per fraction, since 45 Gy plus 5 × 2.65 Gy corresponds to 60 Gy in EQD2 dose. Dose volume histogram cutoff points above described for targets and OARs were compared between the B + I and O-ICBT to show the dosimetric characteristics of $\mathrm{B}+\mathrm{I}$ technique.

High-dose-rate (HDR) brachytherapy treatment plan was performed after the $\mathrm{B}+\mathrm{I}$ dose planning and execu- 
tion time varied from a few minutes to tens of minutes according to the source activity. When HDR brachytherapy treatment finished, the patient was transferred to an accelerator (Synergy ${ }^{\circledR}$, Elekta AB, Stockholm, Sweden) with the applicator still in situ. The IGRT boost was guided by the applicator position on cone beam computed tomography (CBCT). Because the ICBT dose distribution was defined by the position of the applicator, this procedure ensured that the IGRT dose gradients were aligned with the ICBT dose gradients. The applicator was not removed until the IGRT boost finished to minimize any tissue movement and deformation. This procedure was repeated twice a week as scheduled for the conventional brachytherapy treatments performed in our institution.

\section{Results}

It was technically feasible to combine ICBT and IGRT dose distributions. An example is shown in Figure 1. The dose contribution from both the ICBT and IGRT is displayed. The ICBT plan was modified to keep the dose to
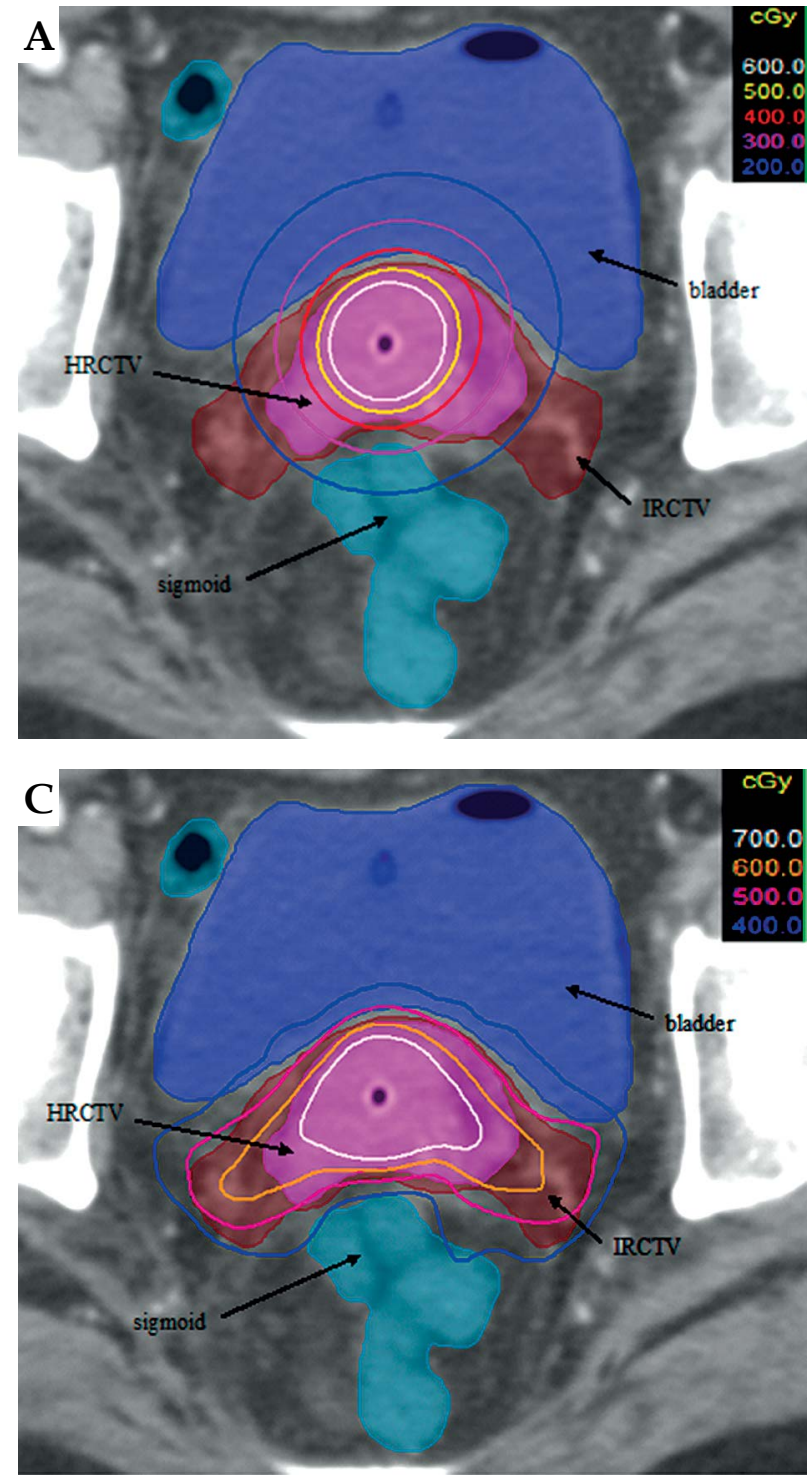

OARs at physical dose constraint while keeping the target covered by the prescription dose as optimal as possible. Image guided radiation therapy boost plan gave dose to the part of the target, which was not covered by the ICBT plan. B + I plan presented substantially better target coverage while keeping the OARs at reasonably low doses.

Table 1 shows mean and standard deviation (SD) in total EQD2 dose delivered by B + I and O-ICBT. For targets, HRCTV mean $\mathrm{D}_{90 \%}$ was comparable for $\mathrm{B}+\mathrm{I}$ and O-ICBT $(p=0.897)$; for $\mathrm{B}+$ I plan, the HRCTV $\mathrm{D}_{100 \%}$, IRCTV $\mathrm{D}_{100 \%}$, and $\mathrm{D}_{90 \%}$ were significantly increased by a mean of $10.52 \mathrm{~Gy}, 5.61 \mathrm{~Gy}$, and 2.70 Gy respectively, if compared with O-ICBT $(p<0.01)$, whereas the O-ICBT delivered higher $\mathrm{D}_{50 \%}$ doses for targets with statistically significant difference. For OARs, bladder, rectum, and sigmoid doses were lower by a mean of 21.36, 6.78 and 10.65 Gy, respectively, for B + I as compared with O-ICBT. With O-ICBT plan, 6, 11, and 11 patients violated $D_{2 c c}$ constraints for the bladder, rectum, and sigmoid, respectively (maximum value 93.35 Gy for bladder, 81.60 Gy for rectum, and 79.64 Gy for sigmoid). V60 Gy

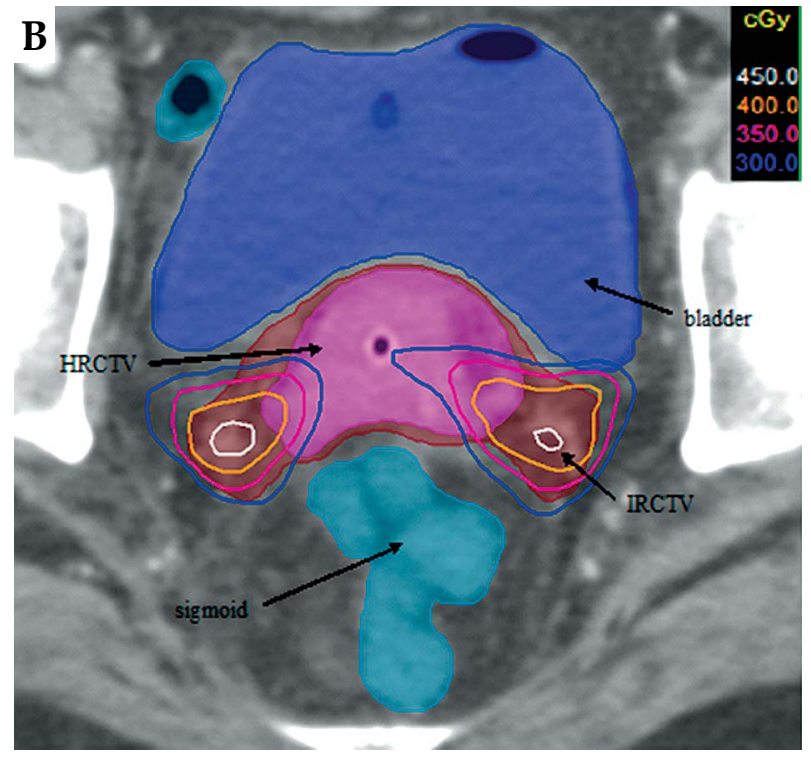

Fig. 1. A sample dose distribution from intracavitary brachytherapy (ICBT) (A), image guided radiation therapy (IGRT) (B), and B + I (ICBT + IGRT) (C) plan for one fraction 
Table 1. EQD2 dose comparison between B + I (ICBT + IGRT) and optimized ICBT (O-ICBT) for targets and OARs for 42 patients. The mean and SD values are listed for each parameter. $P$-value corresponds to pair-wise comparison

\begin{tabular}{|c|c|c|c|}
\hline & $B+1$ & O-ICBT & $p$-value \\
\hline HRCTV $D_{100 \%}$ & 71.01 Gy (1.57) & 60.49 Gy (2.19) & $<0.01$ \\
\hline HRCTV $D_{90 \%}$ & 84.97 Gy (1.65) & 84.90 Gy (0.63) & 0.82 \\
\hline HRCTV $D_{50 \%}$ & 106.29 Gy (5.69) & 114.63 Gy (7.84) & $<0.01$ \\
\hline IRCTV $D_{100 \%}$ & 61.99 Gy (1.18) & 56.38 Gy (1.38) & $<0.01$ \\
\hline IRCTV D $90 \%$ & 76.52 Gy (0.58) & 73.82 Gy (3.04) & $<0.01$ \\
\hline IRCTV $D_{50 \%}$ & 90.69 Gy (2.81) & 99.81 Gy (4.57) & $<0.01$ \\
\hline Bladder $D_{2 c c}$ & 74.73 Gy (2.07) & 86.09 Gy (3.27) & $<0.01$ \\
\hline Rectum $D_{2 c c}$ & 70.12 Gy (2.00) & 72.90 Gy (4.03) & $<0.01$ \\
\hline Sigmoid $D_{2 c c}$ & 70.62 Gy (3.19) & 73.27 Gy (3.58) & $<0.01$ \\
\hline Bladder V60 Gy & $52.84 \mathrm{cc}(8.68)$ & 44.19 cc (6.75) & $<0.01$ \\
\hline Rectum V60 Gy & $19.80 \mathrm{cc}(4.76)$ & 18.63 сc (3.48) & 0.07 \\
\hline Sigmoid V60 Gy & $30.74 \mathrm{cc}(8.86)$ & 19.25 cc (5.39) & $<0.01$ \\
\hline
\end{tabular}

HRCTV - high risk clinical target volume, IRCTV - intermediate risk clinical target volume, $D_{100 \%,} D_{90 \%}, D_{50 \%}$ - the minimum dose received by 100,90 , and $50 \%$ of the prostate volume, $D_{2 c c}$ - minimum dose to the most exposed $2 \mathrm{~cm}^{3}, V 60$ Gy represents the volume irradiated to more than $60 \mathrm{~Gy}$ (EQD2) by the accumulated dose from EBRT and brachytherapy boost

for rectum was almost the same but it was substantially higher for bladder and sigmoid in the B + I plan as compared with the O-ICBT plan. Figure 2 shows typical DVHs for HRCTV, IRCTV, bladder, rectum, and sigmoid for $\mathrm{B}+\mathrm{I}$ and O-ICBT techniques.

\section{Discussion}

Brachytherapy takes advantage of the inverse square law in that high doses of radiation are given to the target and low doses are applied to the healthy tissues. Current use of the tandem ovoid or tandem ring applicator may not adequately cover the clinical target volume for cervical cancer, especially in the parametrial region or in the significant residual tumor site. In this study, we demonstrated that it was technically possible to combine IGRT and HDR ICBT in the treatment of cervical cancer. By adding dose from IGRT to the ICBT dose distribution, $\mathrm{B}+\mathrm{I}$ improved the dose to the target significantly without compromising the constraints for OARs.

Bladder, rectum, and sigmoid are the most sensitive structures to damage during cervical cancer treatment. Georg et al. studied dose effect relationship for late side effects of the rectum and bladder in cervical cancer brachytherapy. They demonstrated that the increase of $\mathrm{D}_{2 \mathrm{cc}}$ in OARs may be translated clinically to an increased probability of late side effects, especially if the $\mathrm{D}_{2 \mathrm{cc}}$ value was close to the specific OARs constraint [19]. In this study, LQ model (EQD2) was used to calculate the targets planning aim dose and OARs dose constraints for B + I. It was theoretically possible to cover target volume while respecting the OARs dose constraints. For cervical cancer patients in our institution, 4 fractions of a boost dose of 5-6 Gy/fraction for HRCTV was recommended. This study highlighted that we can keep OARs doses at rea- sonably low level, treating patients for 5 fractions with a $6 \mathrm{~Gy} /$ fraction dose boost. The mean $\mathrm{D}_{2 \mathrm{cc}}$ for bladder, rectum, and sigmoid was 74.43 Gy, 70.12 Gy, and 70.62 Gy, respectively. Cervical cancer patients with residual malignant cells detected at final stage of brachytherapy may benefit from this technique.

In this study, the HRCTV $\mathrm{D}_{100 \%}$, IRCTV $\mathrm{D}_{100 \%}$, and $\mathrm{D}_{90 \%}$ were significantly improved using $\mathrm{B}+\mathrm{I}$ as compared with O-ICBT. The HRCTV $D_{50 \%}$ is about $25 \%$ higher than $\mathrm{D}_{90 \%}$, which means that the tumor receives an inhomogeneous dose similar to that delivered with ICBT $(35 \%)$, see Table 1. For locally advanced cervical cancer, the advan-

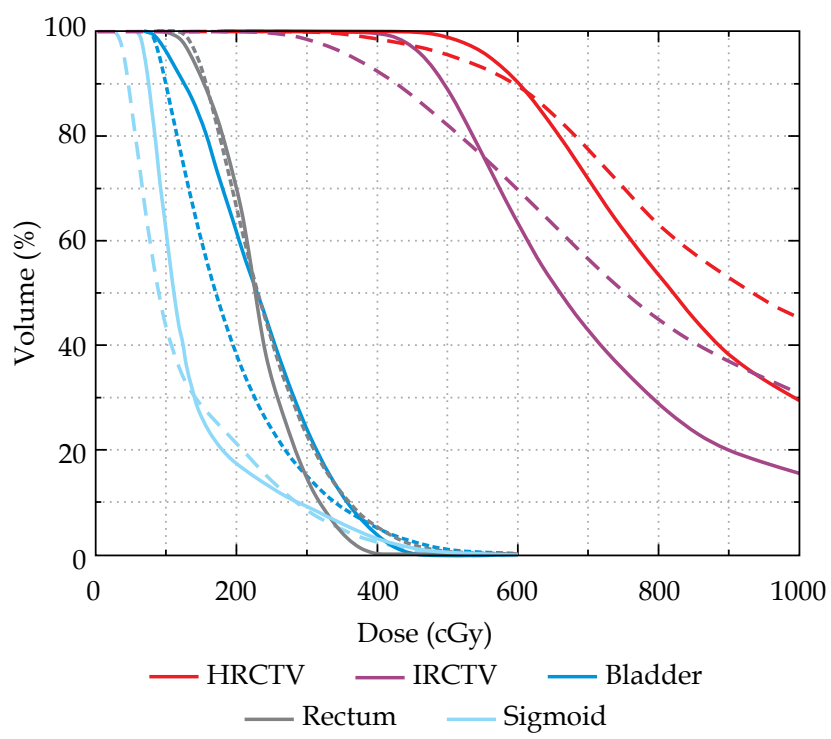

Fig. 2. Comparison of a typical dose volume histograms (DVHs) for B + I (ICBT + IGRT) (solid line) and optimized ICBT (O-ICBT) (dashed line) techniques 
tage of ICBT in the boost is maintained in B + I. For what is concerning OARs, the $\mathrm{D}_{2 \mathrm{cc}}$ standard deviation for bladder, rectum, and sigmoid in B + I was 2.07 Gy, 2.00 Gy, and 3.19 Gy, respectively. We can therefore conclude that the dose to OARs could be controlled within safe limits independently from the tumor location and size.

Parametrial involvement carries poor prognosis; bilateral external beam radiotherapy fields with midline block and IC/IS BT are commonly used to boost parametrial extension [11,20]. Mohamed et al. [21] compared doses delivered by ICBT plus EBRT parametrial boost (PB) with dose delivered by IC/ISBT. With the EBRT PB scenario, patients received lower mean values of HRCTV $\mathrm{D}_{90 \%}$ whereas significantly higher mean values of OARs $\mathrm{D}_{2 \mathrm{cc}}$. However, $\mathrm{B}+\mathrm{I}$ technique is a different way than a simple EBRT PB boost. It overcomes the shortcoming of ICBT and EBRT PB irradiation, and it allows more precise delivery of radiation to the target while sparing normal anatomy compared with conventional radiation. We used the same CT to make the ICBT and the IGRT plan. The IGRT plan was optimized based on the ICBT plan and it was very easy to match the ICBT and IGRT dose components. Applicator guided registration ensured that the IGRT dose gradients were aligned with the ICBT dose gradients. Even with IC/IS BT, the target dose may be insufficient for some large or challenging topography tumors. A study by Assenholt et al. [22] showed that applicator guided IMRT boost in combination with brachytherapy can be used for tumors that are extended over the reach of IC/ISBT applicators.

Studies have investigated the possibility of using IMRT to replace ICBT $[23,24]$. However, IMRT provides a relatively homogeneous dose distribution and cannot provide a dose distribution similar to that of ICBT. The results also show that using an applicator guided IMRT boost instead of ICBT will result in a substantially larger V60 Gy [25]. The clinical impact of increased V60 Gy volume is not known in detail but previous studies have pointed out that a large volume receiving more than $60 \mathrm{~Gy}$ is correlated with side-effects $[26,27]$. It should be mentioned that B + I plan resulted in a larger V60 Gy as compared with the O-ICBT plan, but B + I technique is better than IMRT technique on V60 Gy because the main dose contribution is coming from ICBT and not from IGRT. We still optimize with this technique to enhance the ICBT dose contribution. Before widespread application of B + I in cervical cancer, the effect of low doses to an enlarged area of normal tissue should be evaluated in clinical studies.

Although the current results for B + I techniques for gynecological applications are encouraging, more detailed studies on practical aspects of precision dose delivery need to be performed. Firstly, the cervix is not a fixed pelvic organ, rather one that is subject to movement during treatment $[28,29]$. It was assumed that the target moves together with the applicator and the applicator was fixed to a board so that applicator guided IGRT would minimize setup uncertainties. Secondly, bladder filling has a large impact on the surrounding organs $[30,31]$. In the B + I technique, a bladder catheter was used to control bladder filling, so that the bladder volume was the same during HDR ICBT and IGRT delivery. Thirdly, the IGRT boost plan was delivered immediately after the ICBT with the applicator still in situ, which enabled a precise intersection of IGRT and ICBT dose distributions. Cone beam computed tomography can minimize the uncertainty of patient position; rectum and sigmoid movement was assessed visually on CBCT in as much detail as CBCT image quality allowed.

\section{Conclusions}

It was technically feasible to combine ICBT and IGRT by optimizing the IGRT boost on top of the ICBT dose distribution. The B + I technique not only provides excellent target coverage but also maintains low doses $\left(D_{2 c c}\right)$ to the OARs. It was dosimetrically and logistically feasible to use $\mathrm{B}+\mathrm{I}$ to boost parametrial region or large unfavorable tumor. B + I technique offers an alternative solution to boost very unfavorable topography and extensive disease where the needles may be too challenging to be inserted for full dose coverage. Additional confirmatory prospective studies with larger numbers of patients and longer follow-up are required to validate the durability of these results.

\section{Disclosure}

Authors report no conflict of interest.

\section{References}

1. Lindegaard JC, Fokdal LU, Nielsen SK et al. MRI-guided adaptive radiotherapy in locally advanced cervical cancer from a Nordic perspective. Acta Oncologica 2013; 52: 1510-1519.

2. Nomden CN, de Leeuw AA, Roesink JM et al. Clinical outcome and dosimetric parameters of chemo-radiation including MRI guided adaptive brachytherapy with tandem-ovoid applicators for cervical cancer patients: a single institution experience. Radiother Oncol 2013; 107: 69-74.

3. Gupta P, Aich RK, Deb AR. Acute complications following intracavitary high-dose-rate brachytherapy in uterine cancer. J Contemp Brachytherapy 2014; 6: 276-281.

4. Teke F, Yöney A, Teke M et al. Evaluation of outcome and prognostic factors in 739 patients with uterine cervix carcinoma: a single institution experience. Contemp Oncol (Pozn) 2015; 19: 130-136.

5. Suyama S, Nakaguchi T, Kawakami K et al. Computed tomography analysis of causes of local failure in radiotherapy for cervical carcinoma. Cancer 1998; 83: 1956-1965.

6. Kim RY, Pareek P. Radiography-based treatment planning compared with computed tomography (CT)-based treatment planning for intracavitary brachytherapy in cancer of the cervix: analysis of dose-volume histograms. Brachytherapy 2003; 2: 200-206.

7. Dimopoulos JC, Lang S, Kirisits C et al. Dose-volume histogram parameters and local tumor control in magnetic resonance image-guided cervical cancer brachytherapy. Int $\mathrm{J} R \mathrm{a}-$ diat Oncol Biol Phys 2009; 75: 56-63.

8. Dimopoulos JCA, Pötter R, Lang S et al. Dose-effect relationship for local control of cervical cancer by magnetic resonance image-guided brachytherapy. Radiother Oncol 2009; 93: 311-315.

9. Noyes WR, Peters NE, Thomadsen BR et al. Impact of “optimized" treatment planning for tandem and ring, and tandem 
and ovoids, using high dose rate brachytherapy for cervical cancer. Int J Radiat Oncol Biol Phys 1995; 31: 79-86.

10. Wolfson AH, Abdel-Wahab M, Markoe AM et al. A quantitative assessment of standard vs. customized midline shield construction for invasive cervical carcinoma. Int J Radiat Oncol Biol Phys 1997; 37: 237-242.

11. Okuma K, Yamashita H, Kobayashi R et al. A study of highdose-rate intracavitary brachytherapy boost for curative treatment of uterine cervical cancer. J Contemp Brachytherapy 2015; 7: 128-234.

12. Fenkell L, Assenholt M, Nielsen SK et al. Parametrial boost using midline shielding results in an unpredictable dose to tumor and organs at risk in combined external beam radiotherapy and brachytherapy for locally advanced cervical cancer. Int J Radiat Oncol Biol Phys 2011; 79: 1572-1579.

13. Kirisits C, Lang S, Dimopoulos J et al. The Vienna applicator for combined intracavitary and interstitial brachytherapy of cervical cancer: design, application, treatment planning, and dosimetric results. Int I Radiat Oncol Biol Phys 2006; 65: 624-630.

14. Fokdal L, Tanderup K, Hokland SB et al. Clinical feasibility of combined intracavitary/interstitial brachytherapy in locally advanced cervical cancer employing MRI with a tandem/ring applicator in situ and virtual preplanning of the interstitial component. Radiother Oncol 2013; 107: 63-68.

15. Dimopoulos JC, Kirisits C, Petric P et al. The Vienna applicator for combined intracavitary and interstitial brachytherapy of cervical cancer: clinical feasibility and preliminary results. Int J Radiat Oncol Biol Phys 2006; 66: 83-90.

16. Fokdal L, Tanderup K, Hokland SB et al. Clinical feasibility of combined intracavitary/interstitial brachytherapy in locally advanced cervical cancer employing MRI with a tan$\mathrm{dem} /$ ring applicator in situ and virtual preplanning of the interstitial component. Radiother Oncol 2013; 107: 63-68.

17. De Leeuw AA, Van de Kamer JB, Moerland MA et al. The effect of alternative biological modelling parameters (alpha/ beta and half time of repair T (1/2)) on reported EQD2 values in the treatment of advanced cervical cancer. Radiother Oncol 2011; 101: 337-342.

18. Hellebust TP, Kirisits C, Berger D et al. Recommendations from Gynaecological (GYN) GEC-ESTRO Working Group: Considerations and pitfalls in commissioning and applicator reconstruction in 3D image-based treatment planning of cervix cancer brachytherapy. Radiother Oncol 2010; 96: 153-160.

19. Georg P, Pötter R, Georg D et al. Dose effect relationship for late side effects of the rectum and urinary bladder in magnetic resonance image-guided adaptive cervix cancer brachytherapy. Int J Radiat Oncol Biol Phys 2012; 82: 653-657.

20. Janulionis E, Valuckas KP, Liukpetryte $S$ et al. Californium versus cobalt brachytherapy combined with external-beam radiotherapy for IIB stage cervical cancer: long-term experience of a single institute. J Contemp Brachytherapy 2015; 7: 346-351.

21. Mohamed S, Kallehauge J, Fokdal L et al. Parametrial boosting in locally advanced cervical cancer: Combined intracavitary/ interstitial brachytherapy vs. intracavitary brachytherapy plus external beam radiotherapy. Brachytherapy 2015; 14: 23-28.

22. Assenholt MS, Vestergaard A, Kallehauge JF et al. Proof of principle: Applicator-guided stereotactic IMRT boost in combination with 3D MRI-based brachytherapy in locally advanced cervical cancer. Brachytherapy 2014; 13: 361-368.

23. Aydogan B, Mundt AJ, Smith BD, et al. A dosimetric analysis of intensity-modulated radiation therapy (IMRT) as an alternative to adjuvant high-dose-rate (HDR) brachytherapy in early endometrial cancer patients. Int J Radiat Oncol Biol Phys 2006; 65: 266-273.

24. Wahab SH, Malyapa RS, Mutic S et al. A treatment planning study comparing HDR and AGIMRT for cervical cancer. Med Phys 2004; 31: 734-743.
25. Assenholt MS, Petersen JB, Nielsen SK et al. A dose planning study on applicator guided stereotactic IMRT boost in combination with 3D MRI based brachytherapy in locally advanced cervical cancer. Acta Oncologica 2008; 47: 1337-1343.

26. Barillot I, Horiot J-C, Maingon P, et al. Impact on treatment outcome and late effects of customized treatment planning in cervix carcinomas: baseline results to compare new strategies. Int J Radiat Oncol Biol Phys 2000; 48: 189-200.

27. Huang E-Y, Lin H, Hsu H-C et al. High external parametrial dose can increase the probability of radiation proctitis in patients with uterine cervix cancer. Gynecol Oncol 2000; 79: 406-410.

28. Li XA, Qi XS, Pitterle M et al. Interfractional variations in patient setup and anatomic change assessed by daily computed tomography. Int J Radiat Oncol Biol Phys 2007; 68: 581-591.

29. Kaatee RSJP, Olofsen MJJ, Verstraate MBJ et al. Detection of organ movement in cervix cancer patients using a fluoroscopic electronic portal imaging device and radiopaque markers. Int J Radiat Oncol Biol Phys 2002; 54: 576-583.

30. Ahmad R, Hoogeman MS, Bondar M et al. Increasing treatment accuracy for cervical cancer patients using correlations between bladder-filling change and cervix-uterus displacements: Proof of principle. Radiother Oncol 2011; 98: 340-346.

31. Kim RY, Shen S, Lin H-Y et al. Effects of Bladder Distension on Organs at Risk in 3D Image-Based Planning of Intracavitary Brachytherapy for Cervical Cancer. Int J Radiat Oncol Biol Phys 2010; 76: 485-489. 